\title{
Characterization of Some Graph Classes Using Excluded Minors
}

\author{
Janka Chlebíková *
}

March 28, 2007

\begin{abstract}
In this article we present a structural characterization of graphs without $K_{5}$ and the octahedron as a minor. We introduce semiplanar graphs as arbitrary sums of planar graphs, and give their characterization in terms of excluded minors. Some other excluded minor theorems for 3-connected minors are shown.
\end{abstract}

Keywords: excluded minors, $\leq k$-sum of graphs, tree-width of a graph Mathematics Subject Classification. 05C75, 05C83

\section{Introduction}

Excluded minor theorems characterize the graph classes containing no fixed graph (or graphs) as a minor. The basic excluded minor theorem is known Wagner's reformulation of Kuratowski's theorem: A graph is planar if and only if it has no $K_{5}$ or $K_{3,3}$-minor. The comprehensive overview about known excluded minor theorems can be found in [3] and [11].

The powerful tool for proving excluded minor theorems for 3-connected graphs is the well-known Tutte's Wheel Theorem [12] and its strengthening, Theorem 1.2 below, proved by Seymour [10].

${ }^{*}$ Faculty of Mathematics, Physics and Informatics, Comenius University, Bratislava, Slovakia chlebikova@fmph.uniba.sk 
Theorem 1.1 (Wheel Theorem [12]) Every 3-connected graph can be obtained from a wheel by repeatedly applying operations of adding an edge between two nonadjacent vertices and splitting a vertex.

Theorem 1.2 [10] Let $H$ be a 3-connected minor of a 3-connected graph $G$ such that if $H$ is a wheel, then $H$ is the largest wheel minor of $G$. Then there exists a sequence $H_{0}, H_{1}, \ldots, H_{k}(k \geq 0)$ of 3-connected graphs such that $H_{0}$ is isomorphic to $H, H_{k}$ is isomorphic to $G$, and for $i=1,2, \ldots, k$ the graph $H_{i}$ is obtained from $H_{i-1}$ either by adding an edge between two nonadjacent vertices or by splitting a vertex.

Many excluded minor theorems can be reduced to a simple case checking using Theorems 1.1 and 1.2. This method is demonstrated by Thomas in [11]. Using a similar technique we present some new excluded minor theorems in Section 2. The main result of our research is a structural characterization of graphs without $K_{5}$ and the octahedron as a minor. We also introduce semiplanar graphs as arbitrary sums of planar graphs and give their characterization in terms of excluded minors. We notice that for planar graphs $\leq 3$-sums and $\leq k$-sums $(k>3)$ correspond to the same class of graphs. The presented proof technique can be used to obtain some known results very effectively, which is shown on some examples.

\section{Definitions}

In this paper all graphs are finite and simple. Let $G$ be a graph with vertex set $V(G)$ and edge set $E(G)$. The operation of identifying the endvertices of an edge $e \in E(G)$ and deleting the resulting loop and parallel edges is called contracting the edge $e$. A graph $H$ is a minor of $G$ (or, equivalently said, $G$ has an $H$-minor), if a graph isomorphic to $H$ can be obtained from a subgraph of $G$ by contracting edges. We say that a graph $G$ is obtained from a graph $H$ by splitting a vertex if $H$ is obtained from $G$ by contracting an edge $e$, where $e$ belongs to no triangle in $G$ and both endvertices of $e$ have degree at least three in $G$. By $G \backslash e$ (resp. $G \backslash v$ ) we denote a graph obtained from $G$ by deleting the edge $e$ (resp. the vertex $v$ together with all edges adjacent to $v$ ).

Let $G_{1}$ and $G_{2}$ be graphs of order at least $k+1$. We say that $G$ is a $k$-sum of graphs $G_{1}$ and $G_{2}$, if $G$ is isomorphic to a graph, which can be obtained in the following way: choose cliques $X_{1}$ and $X_{2}$ of the same order $k$ in $G_{1}$ and $G_{2}$, respectively, identify the cliques $X_{1}$ and $X_{2}$ in some way to a single clique and delete some edges (possibly none) of that clique. 0 -sum corresponds to a disjoint union of $G_{1}$ and $G_{2}$. A $k$-sum will be also referred to a $\leq l$-sum for any $l \geq k$. 


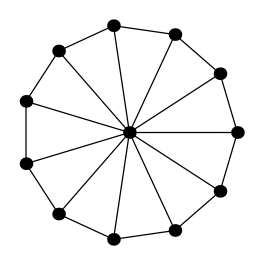

a) $W_{11}$

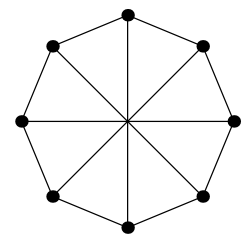

b) $V_{8}$

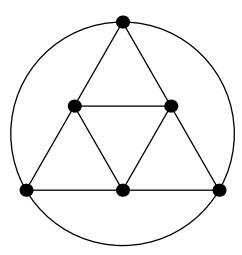

c) the octahedron
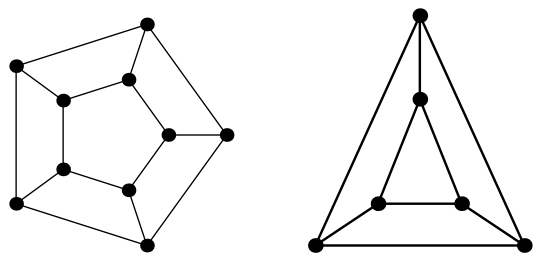

d) $L_{10}$

e) $L_{6}$ (the prism)

Figure 1:

A graph $G$ is internally 4 -connected if it is 3-connected and for every two subgraphs $G_{1}$ and $G_{2}$ of $G$ such that $G_{1} \cup G_{2}=G,\left|V\left(G_{1}\right) \cap V\left(G_{2}\right)\right|=3$ either $\left|E\left(G_{1}\right)\right| \leq 3$ or $\left|E\left(G_{2}\right)\right| \leq 3$.

The wheel $W_{k}(k \geq 3)$ is a graph obtained from a circuit of order $k$ by adding a new vertex joined to every vertex on the circuit (Fig. 1a). By $V_{8}$ we mean a graph obtained from a circuit of order eight by joining every pair of diagonally opposite vertices by an edge (Fig. 1b). The unique 4-connected planar triangulation of order 6 is called the octahedron (Fig. 1c). By $L_{2 k}$ we mean a cyclic planar ladder of order $2 k$ consisting of two vertex-disjoint circuits with vertex sets $\left\{u_{1}, \ldots, u_{k}\right\}$ and $\left\{v_{1}, \ldots, v_{k}\right\}$ (in this order) and edges joining $u_{i}$ and $v_{i}$ for each $i=1,2, \ldots, k$ (Figs. 1d-e).

Notation. Let $G$ be one of the graphs $K_{5}, K_{3,3}$, or $L_{6}$. We denote by $G^{*}$ a unique graph (up to isomorphism) obtained from $G$ by adding an edge between two nonadjacent vertices or splitting a vertex.

\section{New Excluded Minors Theorems}

Firstly, we prove a "meta-theorem", which allows us to focus only on 3-connected graphs:

Theorem 2.1 Let $\mathcal{H}$ be a fixed set of 3-connected graphs and $\mathcal{M}_{\mathcal{H}}$ be the set of all 3-connected graphs with no $H \in \mathcal{H}$ as a minor. Then a graph $G$ has no $H \in \mathcal{H}$ as a minor if and only if $G$ can be obtained by means of $\leq 2$-sums from copies of $K_{1}, K_{2}, K_{3}$, and graphs from $\mathcal{M}_{\mathcal{H}}$.

Proof. The "if" part is easy. If $G$ is a repeated $\leq 2$-sum of graphs having no (3-connected) graph from $\mathcal{H}$ as a minor, then $G$ also cannot have a minor from $\mathcal{H}$. 
To prove "only if" part, assume for the contrary that there exists a graph with no minor from $\mathcal{H}$, which cannot be obtained by means of $\leq 2$-sums from copies of $K_{1}, K_{2}, K_{3}$, and graphs from $\mathcal{M}_{\mathcal{H}}$. Let $G$ be a such graph of the smallest order. Obviously, $G$ is a 2-connected graph of order at least 4 .

Now we prove by contradiction that $G$ is 3-connected. If $G$ is not 3-connected, then there exist two vertices $u, v \in V(G)$ such that $G \backslash\{u, v\}$ is a disconnected graph. Let $G_{1}$ and $G_{2}$ denote two subgraphs of $G$ with the following properties: $\left|V\left(G_{i}\right)\right|<|V(G)|$ (for $\left.i=1,2\right), G_{1} \cup G_{2}=G$ and $V\left(G_{1}\right) \cap V\left(G_{2}\right)=\{u, v\}$. For $i=1,2$ let $G_{i}^{\prime}$ be a graph obtained from $G_{i}$ by adding an edge joining vertices $u$ and $v$ if there is no one (otherwise $G_{i}^{\prime}:=G_{i}$ ). The 2-connectivity of $G$ implies that $G_{1}^{\prime}, G_{2}^{\prime}$ are minors of $G$. Thus, both graphs $G_{1}^{\prime}$ and $G_{2}^{\prime}$ can be constructed from given set of graphs and $G$ is 2-sum of $G_{1}^{\prime}$ and $G_{2}^{\prime}$, a contradiction. Hence, $G$ is necessarily the 3 -connected graph. But $\mathcal{M}_{\mathcal{H}}$ is the set of all 3-connected graphs with no minor from $\mathcal{H}$. It means $G \in \mathcal{M}_{\mathcal{H}}$, a contradiction.

Hence, to describe the structure of all graphs having no graph from a given set of 3-connected graphs as a minor, it is enough to characterize 3-connected graphs with such property. The following lemma contains some basic excluded minor results.

Lemma 2.2 (i) Every 3-connected graph has $K_{4}$ as a minor.

(ii) Every 3-connected graph except $K_{4}$ has $W_{4}$ as a minor.

(iii) Every 3-connected graph which has none of $K_{3,3}, K_{5} \backslash e$, and $L_{6}$ as a minor is isomorphic to a wheel.

(iv) Let $k \geq 5$ be fixed. If a 3-connected graph $G$ has none of $W_{k}, K_{3,3}, K_{5} \backslash e$, and $L_{6}$ as a minor then $G$ is isomorphic to a wheel $W_{l}(l<k)$.

(v) If a 3-connected graph $G$ has $L_{10}$ as a minor, but it contains neither $K_{5}$ nor the octahedron as a minor, then $G$ is isomorphic to $L_{10}$.

(vi) If a 3-connected graph $G$ has $V_{8}$ as a minor, but $K_{5}$ is not a minor of $G$, then $G$ is isomorphic to $V_{8}$.

Proof. The both cases (i) and (ii) follow directly from Theorems 1.1 and 1.2. Furthermore, the case (iii) easily implies the case (iv).

(iii) As follows from Theorem 1.1 every 3-connected graph can be obtained from a wheel $W_{l}(l \geq 3)$ by repeatedly applying the operations of adding an edge 
and splitting a vertex. If $l \geq 4$, then every graph obtained from $W_{l}$ by adding an edge and splitting a vertex has one of the graphs $L_{6}, K_{3,3}$, or $\left(K_{5} \backslash e\right)$ as a minor. If $l=3$, then the graph is isomorphic to a wheel.

(v) Suppose for the contrary that $G$ is not isomorphic to $L_{10}$. As follows from Theorem 1.2, $G$ can be constructed from $L_{10}$ by applying operations of adding an edge or splitting a vertex. But every of three nonisomorphic graphs obtained from $L_{10}$ by adding an edge has either the octahedron or $K_{5}$ as a minor, a contradiction.

(vi) It is easy to see that there are (up to isomorphism) exactly two graphs that can be obtained from $V_{8}$ by adding an edge between two nonadjacent vertices. However, both these graphs have $K_{5}$ as a minor. Hence, if $G$ is not isomorphic to $V_{8}$, then $K_{5}$ is also a minor of $G$ as follows from Theorem 1.2, a contradiction.

In [11] it is proved, that an internally 4-connected nonplanar graph has $K_{5}$ or $V_{8}$-minor, or it is isomorphic to $K_{3,3}$. Due to Lemma $2.2(\mathrm{vi})$, we are able to give a stronger result:

Lemma 2.3 Every internally 4-connected nonplanar graph has $K_{5}$-minor, or it is isomorphic to $K_{3,3}$ or $V_{8}$.

Proof. Let $G$ be an internally 4-connected nonplanar graph with no $K_{5}$-minor, which is not isomorphic to $K_{3,3}$. Then due to the minor properties of internally 4-connected graphs proved in [11], $V_{8}$ is a minor of $G$ and hence $G$ is isomorphic to $V_{8}$ by Lemma $2.2(\mathrm{vi})$.

It is known that every nonplanar 3 -connected graph with no $K_{3,3}$-minor is isomorphic to $K_{5}$ (see [11] or [13]). Applying Theorem 1.2 we obtain the structural characterization of 3-connected graphs with no $K_{3,3}^{*}$-minor.

Theorem 2.4 Every 3 -connected graph without $K_{3,3}^{*}$ as a minor is either a planar graph or isomorphic to $K_{3,3}$ or $K_{5}$.

Proof. Let $G$ be a nonplanar 3-connected graph without $K_{3,3}^{*}$ as a minor, which is not isomorphic to $K_{5}$ or $K_{3,3}$. A nonplanar 3-connected graph with no $K_{3,3}$-minor is isomorphic to $K_{5}$ ([11] or [13]), hence necessarily $G$ has a $K_{3,3}$-minor. According Theorem 1.2 let $H_{0}, H_{1}, \ldots, H_{k}$ be a sequence of graphs applied to $H_{0}:=K_{3,3}$ and $H_{k}:=G$. Since $G$ is not isomorphic to $K_{3,3}$, we see that $k>0$. There is (up to isomorphism) only one possibility for $H_{1}$, namely $K_{3,3}^{*}$. Hence, $K_{3,3}^{*}$ is a minor of $G$, a contradiction. 
Dirac [4] characterizes graphs with no two disjoint circuits (or, in the case of 3 -connected graphs equivalently with no prism as a minor), but his proof is rather complicated. The same result can be deduced easily using Theorem 1.2. We give here a sketch of the proof. Slightly different characterization of graphs with no prism as a minor can be found in [5].

Notation. By $K_{3, k}(k \geq 3)$ we denote the complete bipartite graph. Let $\left\{u_{1}, u_{2}, u_{3}\right\}$ denote nonadjacent vertices of the triple, which are connected with other $k$ vertices. For $i=1,2,3$ let $K_{3, k}^{i}$ denote the graph obtained from $K_{3, k}$ adding $i$ edges between vertices $\left\{u_{1}, u_{2}, u_{3}\right\}$.

Theorem 2.5 Every 3-connected graph without $L_{6}$ as a minor is isomorphic to the one of the following graphs: $K_{5}, K_{5} \backslash e, K_{3, k}, K_{3, k}^{1}, K_{3, k}^{2} K_{3, k}^{3},(k \geq 3)$, or a wheel.

Proof. [Sketch of the proof] As follows from Theorem 1.1 every 3-connected graph $G$ without $L_{6}$ as a minor can be obtained from a wheel $W_{l}(l \geq 3)$ by repeatedly applying operations of adding an edge and splitting a vertex without introducing $L_{6}$ as a minor. For the graph $G$ we introduce two classes of (nonisomorphic) graphs:

$$
\begin{aligned}
\mathcal{N}(G) & =\{H, H \text { is obtained from } G \text { by adding an edge or splitting a vertex }\}, \\
\mathcal{N}_{0}(G) & =\left\{H, H \in \mathcal{N}(G) \text { and } L_{6} \text { is not a minor of } H\right\}
\end{aligned}
$$

Trivially, $\mathcal{N}\left(W_{3}\right)=\mathcal{N}_{0}\left(W_{3}\right)=\emptyset$. Furthermore, it is easy to check that $\mathcal{N}_{0}\left(W_{l}\right)=$ $\emptyset$, if $l \geq 5$, and $\mathcal{N}_{0}\left(W_{4}\right)=\left\{K_{3,3}, K_{5} \backslash e\right\}, \mathcal{N}_{0}\left(K_{5} \backslash e\right)=\left\{K_{5}, K_{3,3}^{1}\right\}, \mathcal{N}_{0}\left(K_{5}\right)=\emptyset$. It can be also verified that for $k \geq 3: \mathcal{N}_{0}\left(K_{3, k}\right)=\left\{K_{3, k}^{1}\right\}, \mathcal{N}_{0}\left(K_{3, k}^{1}\right)=\left\{K_{3, k}^{2}\right\}$, $\mathcal{N}_{0}\left(K_{3, k}^{2}\right)=\left\{K_{3, k}^{3}, K_{3, k+1}\right\}$, and $\mathcal{N}_{0}\left(K_{3, k}\right)=\left\{K_{3, k+1}^{1}\right\}$.

Hence every graph which can be obtained from a wheel $W_{l}(l \geq 3)$ by repeatedly applying operations of adding an edge and splitting a vertex without introducing $L_{6}$ as a minor, is isomorphic to the one of the following graphs: $K_{5}$, $K_{5} \backslash e, K_{3, k}, K_{3, k}^{1}, K_{3, k}^{2} K_{3, k}^{3},(k \geq 3)$.

As easy consequence of the previous theorem and Theorem 1.2 we can prove

Theorem 2.6 Every 3-connected graph without $L_{6}^{*}$ as a minor is isomorphic to the one of the following graphs: $L_{6}, K_{5}, K_{5} \backslash e, K_{3, k}, K_{3, k}^{1}, K_{3, k}^{2} K_{3, k}^{3},(k \geq 3)$, or a wheel. 
Proof. Let $G$ be a 3-connected graph without $L_{6}^{*}$ as a minor.

If $L_{6}$ is not a minor of $G$, then $G$ is isomorphic to one of graphs listed in Theorem 2.5.

If $G$ has an $L_{6}$-minor, then applying Theorem 1.2 to $H=L_{6}$ and $G$ we obtain that $L_{6}^{*}$ is a minor of $G$, a contradiction. Necessarily, $G$ is isomorphic to $L_{6}$.

In the following we introduce the class of semiplanar graphs and give their structural characterization.

Definition 2.7 A graph is semiplanar if and only if it can be obtained from planar graphs by repeatedly applying arbitrary sums.

Theorem 2.8 For a graph $G$ the following statements are equivalent:

(i) $G$ is a semiplanar graph,

(ii) $G$ is a $\leq$ 3-sum of planar graphs,

(iii) $G$ has none of graphs $K_{5}$ and $V_{8}$ as a minor.

Proof. (ii) $\Longrightarrow$ (i) It directly follows from Definition 2.7 of semiplanar graphs.

(i) $\Longrightarrow$ (iii) No $K_{5}$-minor: For the contradiction we assume that $G$ is a semiplanar graph with $K_{5}$-minor such that all semiplanar graphs of smaller order than $G$ have no $K_{5}$ as a minor. Obviously, $G$ is not a planar graph. Due to the minimality of $G, G_{1}$ and $G_{2}$ have no $K_{5}$ as a minor and $G$ is a $\leq 4$ - sum of graphs $G_{1}$ and $G_{2}$. Now it is easy to see, that $G$ has no $K_{5}$ as a minor, a contradiction. $N_{o} V_{8}$-minor: Similarly as in the previous part we suppose that $G$ is a semiplanar graph with $V_{8}$-minor and assume that all semiplanar graphs of smaller order than $G$ have no $V_{8}$-minor. Obviously, $G$ is a nonplanar 3 -connected graph and hence, $G$ is $l$-sum $(l \geq 3)$ of some semiplanar graphs $G_{1}$ and $G_{2}$. Let $G^{\prime}$ denote the graph obtained from $G$ by completing of subgraph $G_{1} \cap G_{2}$ in $G$. $G^{\prime}$ is also the 3 -connected semiplanar graph with no $K_{5}$-minor. But $V_{8}$ is a minor of $G^{\prime}$ and due to Lemma 2.2(vi), $G^{\prime}$ is isomorphic to $V_{8}$. But $G^{\prime}$ contains a triangle, a contradiction.

(iii) $\Longrightarrow$ (ii) Let $G$ be a graph of the smallest order such that $K_{5}$ and $V_{8}$ are not minors of $G$ and $G$ is not a $\leq 3$-sum of planar graphs. Obviously, $G$ is a 3 -connected graph. If $G$ is not internally 4-connected then $G$ is a 3-sum of graphs $G_{1}$ and $G_{2}$. Due to the minimality of $G$, the graphs $G_{1}$ and $G_{2}$ are $\leq 3$-sums of planar graphs and the theorem holds. 
If $G$ is internally 4-connected then $G$ is a planar graph or $G$ is isomorphic to $K_{3,3}$ (Lemma 2.3). This is a contradiction with the assumption that $G$ is not a $\leq 3$-sum of planar graphs.

Wagner [13] proved that a graph has no $K_{5}$-minor if and only if it can be obtained from planar graphs and $V_{8}$ by means of $\leq 3$-sums. In [11] there is another proof of the same result based on the characterization of internally 4connected graphs from Lemma 2.3. In the following lemma we give a short proof of the known characterization of graphs with no $K_{5}^{*}$ as a minor (see [14]) based on the presented proof technique.

Lemma 2.9 For every 3-connected graph $G$ one of the following possibilities appears:

(i) $G$ is $a \leq 3$-sum of planar graphs,

(ii) $K_{5}^{*}$ is a minor of $G$,

(iii) $G$ is isomorphic to $K_{5}$,

(iv) $G$ is isomorphic to $V_{8}$.

Proof. Let $G$ be a 3-connected graph and suppose that $G$ is not a $\leq 3$-sum of planar graphs (case (i)). In such case $K_{5}$ or $V_{8}$ is a minor of $G$ by Theorem 2.8.

If $V_{8}$ is only a minor of $G$, then $G$ is isomorphic to $V_{8}$ by Lemma 2.2 (case (iv)). Now suppose that $K_{5}$ is a minor of $G$ and $G$ is not isomorphic to $K_{5}$. But there is (up to isomorphism) only one graph, denoted $K_{5}^{*}$, which follows from $K_{5}$ in the sequence constructed according Theorem 1.2. It means, $K_{5}^{*}$ is a minor of $G$ (case (ii)).

Theorem 2.10 A graph has no $K_{5}^{*}$-minor if and only if it can be obtained from semiplanar graphs, $K_{5}$ and $V_{8}$ by means of $\leq 2$-sums.

Proof. It follows directly from Theorem 2.1 and Lemma 2.9.

Halin and Jung [6] proved that every 4-connected graph has either $K_{5}$ or the octahedron as a minor. From this result it is easy to prove that every graph with minimum degree at least 4 has $K_{5}$ or the octahedron as a minor. Another selfcontained proof of the previous result can be found in [2]. Maharry [7] presented a characterization of 4-connected graphs without the octahedron as a minor. In what follows we give a structural characterization of graphs which have neither $K_{5}$ nor the octahedron as a minor. 
Definition 2.11 Let $G$ be a graph. Then $G$ has tree-width $k, T W(G)=k$ if $k$ is the smallest integer such that some supergraph of $G$ is $a \leq k$-sum of graphs of order at most $k+1$.

Another definition of tree-width based on a tree-decomposition was introduced by Robertson and Seymour (see [2], or [8] for the proof of its equivalence). Let $T W_{k}$ denote the class of graphs with treewidth at most $k$. For any $k, T W_{k}$, can be characterized by a finite number of excluded (forbidden) minors. The full list of forbidden minors is known only for small values of $k$.

Lemma 2.12 Let $G$ be 3-connected graph which has neither $K_{5}$ nor the octahedron as a minor. Then one of the following holds:

(i) $T W(G) \leq 3$,

(ii) $G$ is isomorphic to $V_{8}$,

(iii) $G$ is isomorphic to $L_{10}$.

Proof. Suppose that $G$ is a 3-connected graph such that $T W(G)>3, G$ has neither $K_{5}$ nor the octahedron as a minor. Then necessarily $V_{8}$ or $L_{10}$ is a minor of $G$, as follows from the known list of forbidden minors for $T W_{3}$ (see [1] or [9]). If $V_{8}$ and $L_{10}$, respectively, is a minor of $G$, then due to Lemma 2.2, $G$ is isomorphic to $V_{8}$ and $L_{10}$, respectively.

Theorem 2.13 A graph has neither $K_{5}$ nor the octahedron as a minor if and only if it can be obtained from graphs with tree-width at most $3, V_{8}$ and $L_{10}$ by means of $\leq 2$-sums.

Proof. It easily follows from Theorem 2.1 and Lemma 2.12.

\section{References}

[1] A. Arnborg, A. Proskurowski, and D. Corneil, Forbidden minors characterization of partial 3-trees, Discrete Math. 80 (1990), 1-19.

[2] J. Chlebíková, Contributions to Algorithmic Graph Theory: Treewidth, Minors, and Balanced Partitions, Ph.D. thesis, Faculty of Mathematics and Physics, Comenius University, Bratislava, January 2000. 
[3] R. Diestel, Graph decompositions. A study in infinite graph theory., The Clarendon Press, Oxford University Press, New York, 1990.

[4] G. A. Dirac, Some results concerning the structure of graphs, Canad. Math. Bull. 6 (1963), no. 2, 183-210.

[5] J. Halin, Über einen graphentheoretischen Basisbegriff und seine Anwendung auf Färbungsprobleme., Diss. Köln (1962).

[6] J. Halin and H. A. Jung, Über Minimalstrukturen von Graphen, insbesondere von n-fach zusammengehägenden Graphen, Math. Ann. 152 (1963), 75-94.

[7] J. Maharry, An Excluded Minor Theorem for the Octahedron, J. Graph Theory 31 (1999), 95-100.

[8] N. Robertson and P. D. Seymour, Graph Minors III. Planar tree-width, J. Combin. Theory Ser. B 36 (1984), 49-63.

[9] A. Satyanarayana and L. Tung, A characterization of partial 3-trees, Networks 20 (1990), 299-322.

[10] P. D. Seymour, Decomposition of regular matroids, J. Combin. Theory Ser. B. 28 (1980), 305-359.

[11] R. Thomas, in Surveys in Combinatorics, (eds. J. D. Lamb and D. A. Preece), Cambridge University Press, Cambridge, 1999.

[12] W. T. Tutte, A theory of 3-connected graphs, Indag. Math. 23 (1961), 441455.

[13] K. Wagner, Über eine Eigenschaft der ebenen Komplexe, Math. Ann. 114 (1937), 570-590.

[14]__ Bemerkungen zu Hadwigers Vermutung, Math. Ann. 141 (1960), $433-451$. 